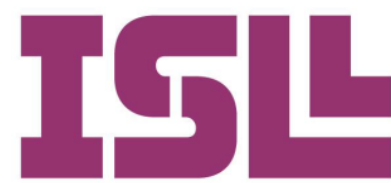

Número 3.

Enero de 2015

\section{Recibido:}

Noviembre 2014

\section{Aprobado:}

Diciembre 2014

Pág. 20 a la 43

\section{Palabras clave}

facilitación de la lectura, legibilidad, mediación lingüística, ELE

\section{Keywords}

facilitation of reading, readability, linguistic mediation, ELE.

\title{
Mediación linguiística del texto literario en la enseñanza del español L2: un estudio sobre las variables de facilitación de la lectura y su legibilidad
}

\author{
Aldo Ocampo González \\ Universidad Los Leones (Chile)
}

\section{Resumen}

Este estudio analiza los procesos de mediación lingüística del texto literario en la enseñanza del español como lengua extranjera. Específicamente, se profundiza en las variables de legibilidad incidentes en la facilitación del discurso escrito a colectivos de estudiantes provenientes de Haití, quiénes reciben clases de español en la ciudad de Santiago de Chile y, demuestran dificultades significativas de acceso a la lectura y a su comprensión. Se opta por un estudio cuantitativo de tipo descriptivo, con un diseño noexperimental de tipo trasversal. Se recurrió a la estadística descriptiva para el procesamiento de los datos recogidos mediante la aplicación de una escala likert dirigida a docentes y estudiantes de español. El primer instrumento describe las estrategias de mediación lingüística utilizadas por el profesorado, mientras que el segundo, efectúa una evaluación de las estrategias de lecturas, gustos y preferencias del estudiantado. Se concluye sobre la identificación de las dimensiones epistémicas que sustentan el discurso de la legibilidad y la lecturabilidad en el campo de la didáctica de segundas lenguas, desconociendo su potencial didáctico y su escaso nivel de implicación en actividades propias del trabajo pedagógico.
Abstract

This study analyzes the processes of linguistic mediation of the literary text in teaching Spanish as a foreign language. Specifically, it explores variables readability incidents in facilitating speech written to groups of students from Haiti, who are taught Spanish in Santiago de Chile and demonstrate significant difficulties in access to reading and understanding. You opt for a quantitative descriptive study with a nonexperimental cross type design. It was used descriptive statistics to process the data collected through the application of a likert scale aimed at teachers and students of Spanish. The first instrument described linguistic mediation strategies used by teachers, while the second, conducting assessment strategies readings, tastes and preferences of the student. We conclude on the identification of epistemic dimensions that underlie speech legibility and readability in the field of second language teaching, ignoring their educational potential and its low level of involvement in activities of the pedagogical work. 


\section{El aprendizaje de la lectura y su legibilidad en la enseñanza del español como L2: ideas para potenciar la comprensión en todos nuestros estudiantes}

Durante la segunda mitad del siglo XX, diversas disciplinas han dirigido sus esfuerzos a comprender el fenómeno lingüístico y sus repercusiones en el proceso educativo. Estas disciplinas podrían agruparse dentro de tres dimensiones: la filosofía analítica o la pragmática filosófica, que aborda el estudio de la actividad lingüística, como parte esencial de la acción humana. En segundo lugar, la antropología lingüística y cultural (Llobera, 1995), la sociolingüística ${ }^{1}$ (Cantero, 1998) y la sociología interaccional $^{2}$ (Widdowson, 1995), que se ocupan de la lengua en relación con los usuarios; entendidos éstos como agentes activos de una comunidad sociocultural determinada. Finalmente, la ciencia cognitiva (Armstrong, 1999; Landívar, 2012), orientada al desarrollo de los procesos y las funciones cognitivas que subyacen a la adquisición y uso de la lectura en el aprendizaje segundas lenguas.

\subsection{La facilitación de la lectura y su legibilidad en la enseñanza del español como L2}

Los enfoques cognitivos sobre el aprendizaje lector, explican diversos factores que se muestran decisivos al momento de observar su efectividad en el aprendizaje de la lectura en ELE. Esta mirada sobre la comprensión de lectura (Gispert y Ribas, 2012), nos invita a profundizar sobre la relación entre lector y texto (Van Esch, 2010).

Estas orientaciones prestan mayor atención a los sistemas de procesamiento de los aprendientes, respecto de las dimensiones de textualidad (Bermeosolo, 2012) que han de superar. En este marco de análisis sobre la comprensión (Perkins, 2003) es entendida como un desempeño creativo (Stone Wiske, 2006) y de naturaleza multiestructural (García y González, 2000).

Asumir la comprensión de textos escritos (Cassany, Luna y Sanz, 1994) como un desempeño creativo y multiestructural, permite considerar las relaciones entre los elementos lingüísticos del mismo y la calidad de las inferencias (Belinchón, Igoa y Riviére, 2007) que de él se desprenden. Numerosas investigaciones (Bryant y Bradley, 1985; De Vega, 1990; Fons, 2004) afirman que esta concepción, establece nuevos sistema de análisis sobre la arquitectura funcional del proceso lector en el campo de la didáctica de segundas lenguas.

\footnotetext{
${ }^{1}$ De acuerdo con el Diccionario de términos claves del Instituto Cervantes (20013), la sociolingüística estudia el lenguaje en relación con la sociedad. Su objetivo de análisis es la influencia que tienen en una lengua los factores derivados de las diversas situaciones de uso, tales como la edad, el sexo, el origen étnico, la clase social o el tipo de educación recibida por los interlocutores, la relación que hay entre ellos o el tiempo y lugar en que se produce la comunicación lingüística.

${ }^{2}$ El "enfoque interaccional" es una "teoría de sistemas" que a su vez forma parte de las teorías de la comunicación. Este enfoque, que analiza las consecuencias pragmáticas de la comunicación interpersonal (entre personas), fue planteado por un grupo de teóricos, biólogos, antropólogos, sociólogos y científicos que pertenecían a la Universidad Invisible de Palo Alto, California. Esta teoría plantea básicamente que la comunicación es un sistema abierto de interacciones que se dan en un entorno o contexto determinado.
} 
Fernández (1999) explica que la comprensión de lectura constituye la meta terminal y tal vez, la más importante del proceso de aprendizaje de una segunda lengua. Según esto, el proceso de lectura y su comprensión involucra una dimensión de tipo lingüística y otra de estilo conceptual o no-lingüística, evidenciando que "la lectura consiste en el dominio simultáneo de las estrategias" (Denyer, 1998, p.31).

La lectura (Calero, 2010; Cuestos, 2011) y su proceso de comprensión (Arroyo, 2013), articula un desafío multidimensional sobre las interacciones, socializaciones y niveles de reflexividad establecidas entre el lector y su contexto (Van Esch, 2010). Para Hymes (1971), esta relación implica la capacidad de cumplir con un repertorio de actos lingüísticos (Arroyo, 2013), que faciliten la integración de los esquemas previos (conocimientos del mundo) de lector y de la nueva información que el texto aporta a esta discusión. García y González (2000), identifican que gracias a este proceso, se incrementa la capacidad de establecer inferencias, comparaciones o simplemente interrogarse a partir de su contenido.

A la luz de estas relaciones, la comprensión de lectura se define en un marco didáctico más amplio, que facilita la integración de la autonomía del lector/aprendiente y la capacidad de reflexionar sobre las fortalezas y debilidades que encuentren en su camino. Deffior (2005), señala que esta mirada fomenta el desarrollo de micro-habilidades que potencian la predicción e inferencias, especialmente desde un enfoque meta-socio-cognitivo (Arroyo, 2009).

Snow (2001), explica que la mejora de la competencia lectora ${ }^{3}$ (Solé, 2012) en la enseñanza del español como L2, queda definida bajo una relación triangular entre lector, texto y actividad (Swartz, 2012). Esta mirada constructivista (Pinker, 1995) y conexionista en su génesis, define la lectura como "un proceso simultaneo de extracción y construcción del significado a través de la interacción e implicación con el lenguaje escrito" (González, 2005, p.32).

En este nuevo marco conceptual, el lector se define como un agente de comprensión. En él se incluyen las experiencias, los conocimientos y las destrezas que este aporta de forma constructiva a su proceso de aprendizaje. Según Molina (1991), el texto es aquello que debe ser comprendido. Esta dimensión involucra una infinidad de procesos mentales, de estructuras textuales y de esquemas de cognición. Esta dimensión considera la estructura y contenido explícito o implícito de la unidad textual.

La actividad constituye parte del desempeño que involucra objetivos, procesos y consecuencias asociadas a la lectura, identificando aquellos factores que

\footnotetext{
${ }^{3}$ Corresponde a la capacidad individual para comprender, utilizar y analizar textos escritos con el fin de lograr sus objetivos personales, desarrollar sus conocimientos y posibilidades y participar plenamente en la sociedad (OCDE, 2009).
} 
favorecen o dificultan su comprensión. Según esta perspectiva, el aprendizaje de la lectura:

(...) es el resultado de la interacción entre procesos de abajo-arriba y arriba-abajo, de forma que la comprensión del texto está dirigida y es el resultado de la interacción simultánea y longitudinal entre los datos proporcionados por el texto y lo que el sujeto aporta al proceso de comprensión (Ramos, 1998, p.35).

La importancia de la comprensión lectora en el enfoque comunicativo se define por la calidad del uso activo que los aprendientes hacen de ésta, en situaciones e interacciones determinadas. Johnson (1981) en sus investigaciones revela el papel crucial que desempeña el contexto como parte de los conocimientos previos del lector sobre el tema del texto. En ellas destaca, que estas interacciones promueven la creación de nuevos desafíos cognitivos que contribuyen a incrementar su experiencia con textos más complejos y diversos.

Wolf (1985), identifica cuatro contextos que resultan fundamentales en el desarrollo y potenciación de la comprensión de lectura en el aprendizaje de una L2, entre los figuran: a) el contexto léxico ${ }^{4}$, b) el contexto sintáctico ${ }^{5}$, c) el contexto semántico $^{6}$ y d) el contexto estilístico. Según esto, la comprensión puede analizarse desde la recepción por parte del lector (proceso dinámico centrado en las condiciones de recepción) y desde el texto como producto de comprensión (enfatizando en las condiciones de producción del mensaje) (Fernández, 1999).

Diversas investigaciones realizadas en el campo de la psicología textual (Mendoza, 2010), confirman la existencia de niveles de representación del discurso (Orlandi, 2012), cada vez más diversos a nivel de estructura mental (Calero, 2010; Cuetos, 2011). Se identifican de este modo, estructuras superficiales y profundas, invitándonos a reflexionar sobre la comprensión como una actividad constructiva se integra de forma holística a las características del texto y en la competencia del lector.

La dimensión textual ${ }^{7}$ contempla las estructuras retóricas de los textos y el análisis de su estructura proposicional ${ }^{8}$ (unidades de inferencia o unidades abstractas de significado) al examinar los procesos de comprensión del discurso escrito. La noción de proposición en la formación del discurso, permite según este autor:

\footnotetext{
${ }^{4}$ Dimensiones encargadas de la transformación de las representaciones ortográficas en conceptos, al procesarlos desde el conocimiento previo almacenado en el léxico interno o lexicón del aprendiente.

${ }^{5}$ Son los responsables del procesamiento de las relaciones sintácticas y gramaticales entre las palabras, un procesamiento que resulta necesario poder construir las proposiciones que relacionan los conceptos descritos como de acceso al léxico.

${ }^{6}$ Aspectos responsables del análisis del contenido conceptual y proposicional de las oraciones, es decir, como así también, del conjunto de operaciones que relacionan unas proposiciones con otras para formar la estructura global de significado que propone la unidad textual.

${ }^{7}$ La dimensión textual de la comprensión lectora, sugiere un análisis sobre las dimensiones vinculadas al desarrollo de las gramáticas de las narraciones defienden, de forma general, que es precisamente este conocimiento el que se activa ante la presencia de un cuento y el que explica la comprensión que tiene lugar, pues funciona como un armazón sobre el que organizar la información del texto (Van Dijk, 1982).

${ }^{8}$ corresponden a unidades discursivas amodales y supramodales (Belinchón, Igoa y Riviére, 2007). Ambas son herramientas fundamentales para la comprensión del discurso. Las inferencias no forman parte del texto de base, pues corresponden al nivel no textual, es decir, al modelo de situación.
} 
(...) explicar los fenómenos tales como la capacidad de reconocer o abstraer la invarianza del significado a pesar de las diferencias de forma que puedan existir entre oraciones sinónimas, el recuerdo del significado y no de materiales literales, los procesos de integración semántica y perdida de información modal en la memoria, y la posibilidad de comparar códigos diferentes, por ejemplo, imágenes y descripciones verbales (Belinchón, Igoa y Riviére, 2007, p.480).

El análisis explícito del contenido, devela un grado de representación más significativo sobre su carácter proposicional (Kintsch y Van Dijk, 1978). Según esto, las proposiciones permiten comprender el carácter oracional del texto y facilitan su significado. Por tanto, comprende más cabalmente la actividad lingüística del aprendiente en su interacción con el texto. De modo que,

(...) la comprensión del discurso parece resultar en varios niveles de representación mental. En esos niveles se incluyen la representación precisa, o literal, de la forma del mensaje, la organización sintáctica de las oraciones, una red integrada de proposiciones subyacentes al mensaje, y un modelo de la situación a la que éste se refiere (Singer, 1990, p.27).

La comprensión desde el punto de vista de la estructura cognitiva depende del sistema de modelamiento que la situación textual realice, permitiendo la construcción de un modelo basado en redes semánticas y en los procesos de tipo conexionistas (Kintsch, 1988). Frente a esto: ¿qué significa comprender un texto?, ¿qué rol juegan las estructuras discursivas y sus representaciones? o ¿qué aporta a esta discusión el estudio cognitivo del discurso en el campo de la didáctica de la lectura en L2? En respuesta a estas interrogantes, se afirma que

(...) el tratamiento que el lector hace del texto está determinado por características propias del lector (experiencia personal, interés por el tema), de tal suerte que, para que el sujeto logre mejorar su lectura, se requiere tener en consideración las estrategias óptimas para el desarrollo del pensamiento que permitan estructurar de manera lógica la información. Principalmente se abordan los procesos de análisis y síntesis; el primero para conocer los diferentes elementos que integran el escrito, y el segundo para descubrir las relaciones entre los elementos y establecer los juicios (Acosta, 1989, p.72).

Según esto, será el lector quién construya los significados del texto a partir de sus experiencias, esquemas y motivaciones. Entonces la compresión resultaría producto de la negociación entre texto y lector en un contexto determinado, contribuyendo a reconocer la importancia de los géneros textuales que utilizamos en múltiples situaciones de la vida social cotidiana. La facilitación del discurso escrito se asume como una herramienta de transformación de las prácticas de enseñanza ligadas al desarrollo de la lectura en la enseñanza del español como L2, pues permite replantear las bases didácticas asociadas a las diversas formas de interacción entre el lector, el texto y su contexto ${ }^{9}$.

${ }^{9}$ Corresponde a la situación inmediata que envuelve al acto de habla (Mendoza, 2010). 
La lingüística del texto y del discurso, nos invita a reflexionar acerca del significado oculto que hoy encierra la idea de texto. Numerosos autores (Alonso y Mateos, 1985; Calero, 1997; Mendoza, 2001) abordan la relación texto-discurso, incorporando en una denominación común, las diversas huellas enunciativas del hablante (Benveniste, 2007), a través de una unidad ideológica con sentido transformacional (Orlandi, 2012). Así, el texto es definido como "una construcción teórica abstracta que subyace a lo que normalmente se llama discurso" (Van Dijk, 1993, p.86).

Brown y Yule (1990), explican lingüística textual como un sistema de disposiciones capaces de abordar la unidad a partir de la actividad o del uso activo de la lengua, lo que se compondría al menos, de "una oración, que posee textualidad, y que se manifiesta en forma de discurso" (Dressler y Beaugrande, 1981, p.52). Para la gramática sistémica funcional (Adams, 1980), la capacidad de textualidad se organizaría a unidades de cohesión (Halliday y Hassan, 2000), cada vez más profundas y diversificadas.

La legibilidad involucra actividades y estrategias, que intentan bajo un modelo lingüístico-textual, dispone de estrategias para facilitar la comprensión y el significado construido por el lector. Los mecanismos de facilitación del discurso escrito o de la lectura, existirían a partir de un texto existente, cuyo potencial lingüístico-discursivo (Cassany, 1993; De Arriba, 2003) y pedagógico (Freinet, 1973), justificaría su adaptación acorde al perfil lingüístico y cultural del aprendiente. Resulta relevante entonces, efectuar una evaluación contextualizada sobre el texto, la actividad y los criterios de flexibilización para promover la comprensión en todos y cada uno de nuestros estudiantes (Ocampo, 2014).

Investigaciones recientes (Inchaurrable y Vázquez, 2000) señalan que la construcción del conocimiento declarativo en las interacciones áulicas y lectoras, plantean la necesidad de redefinir el papel de los conocimientos previos y su relación con la comprensión lectora (Calero, 2010). En términos generales, la concepción cognitiva y constructivista del aprendizaje ha demostrado en la última década el cuidado que debe prestarse al papel de los conocimientos previos, entendiendo que su construcción dependen únicamente de la calidad de la experiencia lingüística y de la pertinencia de su socialización.

Leer supone de este modo:

(...) enfrentar nuestros conocimientos con los que proporciona el texto, es decir, flexibilizar ambas realidades con una enorme dosis de construcción personal, donde ésta última, se ve mediada por nuestras representaciones mentales que viene siendo algo así como la interpretación mental del acontecimiento o simplemente la apropiación del significado (Gil, 2001, p.48).

La enseñanza de la lectura desde un enfoque comunicativo, permite atender a la comprensión en uso y a los sistemas de representación desarrollados por los estudiantes 
(Solé, 1992). Autores como Smith y Van Dijk, señalan a la luz de sus investigaciones que la destreza lectora, sólo cobrará sentido a nivel de competencia comunicativa y cognitiva de los aprendientes, sólo si se consideran los conocimientos previos o conocimientos del mundo por parte del lector. Por tanto, los textos auténticos contribuirían a mediar más pertinentemente la teoría acerca del mundo y el sentido del texto con nuestros conocimientos previos (Smith, 1984).

\subsection{Variables lingüísticas para la determinación del nivel de legibilidad en la enseñanza de la lectura del español como L2}

\begin{tabular}{|c|c|c|}
\hline \multicolumn{3}{|c|}{$\begin{array}{c}\text { Criterios para promover la legibilidad, comprensión lectora y control de lectura en la enseñanza del } \\
\text { español como L2. Anula, } 2005 .\end{array}$} \\
\hline Nivel. & Proceso de control. & Proceso de control. \\
\hline $\begin{array}{l}\text { Nivel } \\
\text { Léxico- } \\
\text { fonológico. }\end{array}$ & $\begin{array}{l}\text { - Control de la extensión } \\
\text { de las palabras. } \\
\text {-Control de la } \\
\text { complejidad } \\
\text { silábica de } \\
\text { las palabras }\end{array}$ & $\begin{array}{l}\text {-Cambiar las palabras excesivamente extensas, medida en número de } \\
\text { sílabas, por otras más breves. } \\
\text {-En las sustituciones léxicas, elegir, en la medida de lo posible, palabras } \\
\text { cuyo patrón silábico sea de los de mayor frecuencia del español. }\end{array}$ \\
\hline $\begin{array}{l}\text { Nivel léxico- } \\
\text { semántico. }\end{array}$ & $\begin{array}{l}\text {-Control de las palabras } \\
\text { abstractas } \\
\text {-Control de la frecuencia } \\
\text { léxica } \\
\text {-Control de la reiteración } \\
\text { léxica y la sinonimia } \\
\text { léxica. }\end{array}$ & $\begin{array}{l}\text {-Utilizar palabras que sean de uso actual y de alta frecuencia. } \\
\text {-Sustituir los conceptos abstractos por conceptos concretos. } \\
\text {-Repetir las mismas palabras para los mismos conceptos (especialmente } \\
\text { para las palabras de frecuencia baja o moderada). Reducir la diversidad } \\
\text { léxica del texto. }\end{array}$ \\
\hline $\begin{array}{l}\text { Nivel léxico- } \\
\text { semántico }\end{array}$ & $\begin{array}{l}\text {-Control de los préstamos } \\
\text { Léxicos. } \\
\text {-Control de siglas y } \\
\text { Acrónimos. }\end{array}$ & $\begin{array}{l}\text { Evitar los neologismos procedentes de otras lenguas, } \\
\text { los extranjerismos, así como los términos jergales. } \\
\text { Evitar las abreviaturas, iniciales, acrónimos, etc. }\end{array}$ \\
\hline $\begin{array}{l}\text { Nivel flexivo } \\
\text { / verbal. }\end{array}$ & $\begin{array}{l}\text {-Control de las } \\
\text { construcciones } \\
\text { Perifrásticas. } \\
\text {-Control de la } \\
\text { variabilidad flexiva } \\
\text { verbal. } \\
\text {-Control de la extensión } \\
\text { Oracional. } \\
\text {-Control de la } \\
\text { complejidad } \\
\text { Sintáctica. } \\
\end{array}$ & $\begin{array}{l}\text {-Evitar la variabilidad de formas verbales. } \\
\text {-Reducir la presencia de tiempos compuestos o complejos, así como la } \\
\text { presencia de construcciones de subjuntivo. } \\
\text {-Evitar las construcciones perifrásticas } \\
\text { Procurar que las oraciones sean breves, esto es, que no superen las quince } \\
\text { palabras por oración. } \\
\text { Evitar las estructuras sintácticas complejas, tales como oraciones que } \\
\text { contengan más de dos corchetes oracionales, por ejemplo: [O.ppal. } \\
\text { [O.sub.1 [O.sub.2 [O.sub.3]]]]. Evitar las estructuras parentéticas: } \\
\text { incisos, circunloquios, etc. }\end{array}$ \\
\hline Nivel Textual. & $\begin{array}{l}\text { - Control de la coherencia } \\
\text { y la cohesión } \\
\text { del texto }\end{array}$ & $\begin{array}{l}\text {-Garantizar que el texto sea coherente (pueda ser entendido como una } \\
\text { unidad) y esté cohesionado mediante una adecuada trabazón lingüística } \\
\text { en la que se primen los procedimientos basados en la recurrencia y el uso } \\
\text { de marcadores textuales que organicen el discurso y marquen } \\
\text { adecuadamente las relaciones lógicas entre las distintas oraciones y } \\
\text { frases. }\end{array}$ \\
\hline $\begin{array}{l}\text { Nivel } \\
\text { Conceptual. }\end{array}$ & $\begin{array}{l}\text {-Control de los elementos } \\
\text { referenciales } \\
\text {-Control de la densidad } \\
\text { proposicional o } \\
\text { predicativa. }\end{array}$ & $\begin{array}{l}\text {-Garantizar que sea correctamente identificada la referencia de los } \\
\text { participantes en el discurso. } \\
\text {-Evitar que las oraciones transmitan numerosas proposiciones mediante } \\
\text { el control del número de predicados por oraciones y de los elementos con } \\
\text { capacidad predicativa propia. Crear mensajes de una idea por oración. }\end{array}$ \\
\hline
\end{tabular}

Tabla 1: Dimensiones e Indicadores de Legibilidad, Comprensión lectora y Control de Lectura. Tomado del Texto: "Introducción al Quijote de la Mancha de Fácil Lectura". Fuente: Anula, Fernández-Lagunilla, Belinchón y Revilla, 2005:25. 


\section{Objetivos de Investigación}

3.1. Conocer los procesos de mediación lingüística vinculados a la utilización del texto literario como recurso didáctico en la facilitación de la lectura y su legibilidad en la enseñanza del español como lengua extranjera.

3.2. Analizar los criterios y niveles de adaptación lingüística y discursiva, respecto de las estrategias de mediación lingüística del texto literario como recurso didáctico en la enseñanza de la comprensión lectora en aprendientes de español como L2.

3.3. Describir el impacto que tienen las estrategias didácticas utilizadas al desarrollar las micro-habilidades de comprensión lectora mediante la utilización del texto literario como recurso didáctico, respecto del desarrollo de la competencia comunicativa y sus sub-competencias en aprendientes de español como L2.

\section{Metodología}

\subsection{Enfoque y diseño}

El estudio se encuadra bajo los lineamientos cuantitativos de investigación (Bunge, 1980; Pérez, 1998), asumiendo un enfoque de tipo no experimental descriptivo del tipo de encuestas. El diseño descriptivo "describe cómo es y cómo se manifiesta determinado fenómeno en la realidad” (Hernández, 2003:60). Desde esta perspectiva, los estudios descriptivos pretenden especificar propiedades importantes de comunidades, grupos o cualquier fenómeno que sea sometido a análisis (Hernández, 2003).

El diseño metodológico (Giddens y Turner, 2010) recogido en esta investigación se encuadró en un sistema de tipo no-experimental (Sousa, Driessnack y Costa, 2007) de carácter transversal o transaccional (Hernández, 2003). Estos diseños pueden definirse como aquellas investigaciones que se realizan sin manipular deliberadamente las variables ${ }^{10}$, permitiendo sólo la observación de los fenómenos en su ambiente natural para después ser analizados. Según su temporalización, esta asumió un carácter transaccional ya que los datos se recolectan en un solo momento, en tipo único, intentando describir las variables participantes e interpretar su incidencia e interrelación en un momento determinado (Hernández, 2003).

\subsection{Muestra}

A través de un muestreo no-probabilístico de tipo disponible, el cual incluye en la muestra a los sujetos de investigación de acuerdo a su disponibilidad (Cardona,

${ }^{10}$ Una variable es una propiedad que tiene una variación que puede medirse u observarse. 
2002). La encuesta fue aplicada a una muestra bietépica compuesta por docentes y estudiantes de español pertenecientes a un centro de idiomas ubicado en la ciudad de Santiago de Chile, Región Metropolitana.

La población de investigación quedó conformada por un $\mathrm{N}=12$ sujetos de investigación, de los cuales el 33.3\% corresponde a docentes que imparten clases de español en dicho laboratorio de idiomas, cuyas edades fluctúan entre 29 a 37 años. Según la participación de los docentes de español como L2, se identifica que un 25\% de ellos son de sexo masculino y un $75 \%$ de sexo femenino. De acuerdo a lo informado, según su experiencia laboral, un $50 \%$ de los mismos posee entre 3 a 5 años de ejercicio profesional, mientras que un $25 \%$ asegura tener entre 11 a 15 años de trabajo. Sólo el 25\% restante se ubica entre los 16 a 20 años de ejercicio y/o experiencia profesional.

Un $67.7 \%$ de los sujetos de investigación corresponde a los estudiantes de español provenientes de la República de Haití, quienes por diversas razones de movilidad social y cultural residen en Chile, sus edades fluctúan entre los 26 a 42 años. Un $62.5 \%$ de los aprendientes de español se ubican en un nivel B2 ${ }^{11}$, según los datos arrojados por la evaluación interna aplicada a cada aprendiente. Este instrumento recoge las orientaciones propuestas por el Plan Curricular del Instituto Cervantes.

Sólo un $37.5 \%$ de los sujetos seleccionados para esta investigación se ubican en un nivel $\mathrm{A} 2^{12}$. Se observa que un $38 \%$ de ellos corresponden a sexo masculino, mientras que un $62 \%$ de sexo femenino. Se observa que un $50 \%$ de los aprendientes reside en la ciudad de Santiago de Chile entre 2 a 3 años, un $13 \%$ declara un período de residencia que oscila entre 4 a 5 años y, un $37 \%$ de los mismos, afirma un tiempo de residencia menos a 1 año en este país.

\subsection{Instrumentos de Investigación}

La técnica de recolección de información utilizada para este estudio, corresponde a una escala tipo Likert ${ }^{13}$ (Follari, 2007) dirigida a profesores y aprendientes de español. La escala dirigida a los docentes de español, denominada

${ }^{11}$ La etapa o estadio B1 y B2 define la capacidad de usuario independiente. No obstante, el Plan Curricular del Instituto Cervantes (2006), explica que los aprendientes de ubicados en un nivel B1, disponen de los recursos lingüísticos y no lingüísticos necesarios para participar en los intercambios comunicativos con un grado de fluidez, precisión y naturalidad suficientes como para que los interlocutores no tengan que hacer un esfuerzo especial.

${ }^{12}$ De acuerdo con el MCER (2002) en su dimensión de agente social, se explica que el nivel inicial contempla el marco A1 y A2 definiendo una capacidad lingüística a nivel de usuario. Según el Plan Curricular del Instituto Cervantes (2006), los estudiantes que alcanzan este nivel son capaces de manejar un repertorio limitado de recursos linguiísticos y no lingüísticos sencillos, como estructuras sintácticas básicas (que incluyen frases o formulas memorizadas) y conocimientos muy generales sobre convenciones sociales y referentes culturales del mundo hispano.

${ }^{13}$ Instrumentos de medición utilizada en la investigación socioeducativa para describir el grado de actitud o disposición de los encuestados. 
Cuestionario A.1: "Escala sobre de Estrategias de mediación lingüística y didáctica en el marco de la facilitación de la lectura a estudiantes haitianos", compuesto por un total de 19 enunciados, de tipo autoadministrado, la cual fue proporcionada de forma directa e individual a cada sujeto de investigación con responsabilidad docente.

Este instrumento se compone de 3 dimensiones, tales como: a) criterios de facilitación de la lectura según el MCER y PCIC (con un $15.78 \%$ de enunciados), b) estrategias de mediación lingüística (con un $63.15 \%$ de enunciados) y c) el texto literario como recurso didáctico (con un $21.5 \%$ de enunciados). Su puntaje máximo es de 76 puntos, lo que demuestra una actitud favorable acerca de la utilización de estrategias de mediación linguiística como recurso de potenciación de la lectura en estudiantes de español como L2, mientras que su puntaje mínimo corresponde a 19 puntos, reflejando una actitud menos favorable ante del desarrollo de tales dispositivos pedagógicos en materia de aprendizaje y desarrollo lector.

El propósito de este instrumento es conocer las estrategias de mediación linguiística y de facilitación de la lectura, respecto de los sistemas didácticos desarrollados por los profesores de español como elemento de diversificación de la enseñanza a estudiantes de procedencia haitiana residentes en la ciudad de Santiago de Chile.

El instrumento dirigido a los aprendientes de español como L2, denominado Cuestionario A.2: "Escala sobre evaluación de estrategias de lectura, gustos y preferencias en aprendientes de español como L2", compuesto por un total de 23 enunciados, de tipo autoadministrado, la que fue proporcionada de forma directa e individual a cada uno de los aprendientes de español como L2.

Este instrumento se compone de 3 dimensiones, tales como: a) preferencias y gustos en el proceso lector (con un $21.5 \%$ de enunciados), b) evaluación de estrategias de lectura (con un $63.15 \%$ de enunciados) y c) procedimientos y técnicas de aproximación al texto (con un $26.31 \%$ de enunciados). Su puntaje máximo es de 92 puntos, lo que demuestra una actitud favorable hacia el desarrollo de estrategias de lectura y las preferencias desarrolladas por los aprendizajes en este campo. Su puntaje mínimo corresponde a 23, reflejando una actitud menos favorable ante gustos y preferencias en materia de lectura como proceso de desarrollo social y cultural de cada aprendiente participante de la investigación.

El propósito de esta escala consiste en explorar las concepciones de estudiantes provenientes de Haití, respecto de la utilización de textos literarios en la enseñanza del español como L2, así como sus hábitos, gustos y preferencias de lectura, respecto de sus variantes de procesamiento. En cada una de los enunciados de ambos instrumentos se identifican cuatro criterios, a saber: totalmente de acuerdo (4), 
de acuerdo (3), en desacuerdo (2) y totalmente en desacuerdo (1), debiendo cada participante seleccionar la variable o criterio que más le identifique.

La validación del instrumento se realizó mediante la técnica de jueces calificados a nivel nacional e internacional, coincidiendo todos los expertos en que el instrumento presentado posee validez de constructo, de contenido y consistencia interna (fiabilidad).

\subsection{Plan de Análisis de la Información}

Los resultados fueron analizados e interpretados de modo descriptivo y analítico, recurriendo a la estadística descriptiva (McMillan y Schumacher, 2007), para delinear la distribución de los resultados obtenidos en la escala.

De tal forma, para obtener tales representaciones se empleó la distribución de frecuencia, distribución porcentual, cálculo de media, moda y mediana aritmética. Finalmente, la posterior tabulación de datos, elaboración de tablas de contingencia y gráficos se efectuó a través de la utilización del programa SPSS.

\section{Resultados de Investigación}

\subsection{Análisis de Resultados referidos a Escala Likert dirigida a Docentes de Español como L2}

\subsubsection{Criterios de Facilitación de la Lectura en la enseñanza del español como L2}

En la apreciación general se observó que un 75\% de los docentes señalan estar totalmente de acuerdo con la integración de estrategias de simplificación de la lectura más oportunas y pertinentes al perfil lingüístico de sus aprendientes. Se reconoce entonces, la necesidad de avanzar sobre el desarrollo de propuestas teóricas y metodológicas capaces de contribuir a re-pensar la enseñanza de la lectura y su promoción al interior de los dispositivos de formación en el campo de las didácticas de segundas lenguas.

Un $65 \%$ enfatiza sobre la necesidad de asumir la enseñanza de la lectura desde parámetros que integren los principios de la diversificación didáctica como elemento de compensación de las desigualdades educativas y cognitivas. El desafío es hoy, llegar a todos nuestros estudiantes, a través de nuevas concepciones didácticas que efectivamente brinden mayores espacios de accesibilidad al aprendizaje de la lectura en su lengua meta y, de este modo, se operacionalicen los derechos en la educación.

Según esto, la facilitación del discurso escrito demuestra un potencial relevante y significativo en la gestión de nuevas oportunidades socioeducativas en 
materia de promoción y desarrollo de la lectura. Se contribuye desde esta perspectiva, a romper con los tradicionales universalismos negadores de las diferencias, que en materia de lectura, exigen la presentación de una sola versión a un amplio colectivo de estudiantes, cada vez más heterogéneos.

Esta visión más centrada en el aprendizaje y en las potencialidades del sujeto, bajo la concepción de sujeto en derecho, nos invita a cuestionar los criterios de selección utilizados en la enseñanza del español para promover el óptimo aprendizaje y dominio lector de la lengua. Todo ello, nos invita a superar la simple adaptación de textos, sino avanzar hacia nuevas formas de comprensión sobre el proceso lector y sus variantes de legibilidad insertas, especialmente a nivel de sus estructuras discursivas.

Se asume entonces, que la facilitación de la lectura y sus implicancias pedagógicas, intentan articular un conocimiento situado (Zemelman, 2012) que avanza por sobre las conceptualizaciones tradicionales sobre el aprendizaje y el desarrollo lector en segundas lenguas. Esta aproximación, implica reconsiderar el texto y asegurar nuevas formas de conocimientos lingüísticos en cada aprendiente en cada interacción con este.

En particular, los docentes comprenden este proceso como una actividad comunicativa básica, equiparable a actividades de recepción, interacción y producción, permitiendo enfatizar en la modificación estructural (lingüística y discursiva) del texto, en sus dimensiones didácticas y formativas. Sin duda, el desafío es hoy, promocionar nuevas formas de comunicación didáctica al interior del aula de español como segundas lenguas. Todo ello, nos obliga a pensar si efectivamente las condiciones formativas y/o didácticas inscriptas en el campo de las segundas lenguas pertinente asegurar la comprensión como una capacidad multidimensional y diversificada a partir de cada una de las dimensiones cognoscitivas propias del aprendiente.

No obstante, el 50\% de los encuestados señala que el Marco Común Europeo de Referencia promueve a nivel discursivo y de propuesta didáctica la facilitación de la lectura en el campo de la didáctica de segundas lenguas. Sólo así, el 25\% de ellos afirma que el sustrato teórico que vertebra el campo de la facilitación de la lectura en la enseñanza del español como lengua extranjera intenta promover la universalización de la lectura como medio de equiparación y compensación de las desigualdades educativas. Por tanto, ¿qué condiciones estructurales, pedagógicas, cognitivas e ideológicas debe considerar la propuesta de universalización de la lectura?, ¿será entonces, la universalización de la lectura una nueva forma de homogenización social en materia de acceso y promoción de la lectura? 
Actualmente, existen dos enfoques destinados a superar las desigualdades sociales y educativas, estos son, el enfoque de educación inclusiva y el de educación para todos. Hasta ahora, las categorías de diversidad han sido asumidas como parte de los diferentes, obedeciendo así a una construcción social y quitando al ser humando su principal propiedad que lo define como humano, es decir, como sujeto variable.

Pensar un sistema de universalización basado en una concepción de totalidad, implica asumir nuevas formas de significar a un conglomerado amplio de estudiantes bajo una premisa cuyos efectos de poder van más allá de un simple acto semántico. El tiempo histórico que nos toca vivir, cuestiona profundamente el entendimiento de estas categorías, invitándonos en palabras de Enrique Dussel a asumir una visión analéctica $^{14}$ sobre los mismos, a fin de ir más allá de la totalidad y efectivamente encontrarse con el otro y la otra. De todos modos, será relevante reconocer si efectivamente existe otro u otra en la producción de figuras de alteridad que hoy delineamos bajo el discurso promocional de la educación inclusiva y educación para todos.

Sin duda, esta mirada nos invita a tensionar la idea de totalidad, pues desde un análisis analéctico estaríamos reproduciendo una nueva forma de homogenización encubierta bajo un discurso esperanzador y contradictorio en sus formas de realización prácticas (referido a las diversas áreas de trabajo pedagógico). Por tanto, la contribución de una matriz epistémica en materia de legitimación de la diversidad y de educación para todos, debe ante todo, clarificar, re-significar y re-pensar el devenir de sus conceptos basales en función de las demandas, necesidades y motivaciones que emergen a partir de la multiplicidad de observaciones ciudadanas. Todo ello, de alguna u otra forma también tiende a generar nuevas formas de exclusión y medidas de pseudo-inclusión en el acceso, promoción y aprendizaje de la lectura, especialmente, a colectivos de ciudadanos no hablantes de español.

Un dato relevante dentro de esta discusión, es que los docentes (50\%) perciben que los lineamientos expuestos por el Marco Común Europeo de Referencia y el Plan Curricular del Instituto Cervantes consideran los saberes culturales pertinentes a las necesidades lingüísticas del español en América Latina bajo el enfoque de facilitación del proceso lector dentro de la región (25\%).

De esto se desprende que un $25 \%$ explica estar totalmente en desacuerdo con que el proceso de facilitación del discurso escrito adquiera únicamente un mecanismo de simplificación lingüística y sintáctica puede entenderse con una variante de traducción o interpretación del texto que busca ser facilitado, mientras que el $25 \%$ restante se muestra totalmente de acuerdo con esta afirmación.

${ }^{14}$ Concepto acuñado por Juan Carlos Scannonne posteriormente profundizado por Enrique Dussel en la Filosofía de la Liberación. 
Sin duda que este nivel de discusión permite inferir que las temáticas ligadas a la facilitación del discurso escrito y sus variantes implícitas en el desarrollo de la competencia comunicativa y discursiva; resultan escasamente comprensibles en cuanto a los beneficios, desafíos y oportunidades sociales, culturales y educativas que éstas suponen para los aprendientes de español y en particular; para aquellos provenientes de la República de Haití. Por tanto, el desafío esta no sólo en promover de marcos conceptuales teóricos, metodológicos y prácticos en materia de acceso y promoción de la lectura, sino que concepciones tendientes a operacionalizar los derechos al interior de la estructura social y así, cumplir la tan anhelada inclusión social de muchas personas que por diversas razones no logran comprender diversos documentos, especialmente, los que dicen relación con las leyes, contratos laborales, ente otros.

De este modo, la educación debe ser cada vez más cercana al ser humano y esto, representa uno de los puntos críticos que no hemos sido capaces de resolver aún. A modo de conclusión, Montessori señala, "sólo podemos darle a cada individuo la oportunidad de satisfacer sus potencialidades para que éste sea un ser humano independiente, seguro y equilibrado" (Montessori, 1934; citado en Landívar, 2013, p.27).

\subsubsection{Estrategias de Mediación Linguiística y potenciación del desarrollo lector en español como L2}

Al consultar a los docentes sobre el nivel de conocimientos que éstos poseen sobre las variables discursivas y lingüísticas implicadas en el proceso de facilitación de un texto literario en la enseñanza del español como L2, sólo un 75\% afirmó estar afirmo estar en conocimiento de ellas, especialmente a partir de las clásicas medidas aportadas por el campo de la mediación lingüística provenientes de las ciencias de la traducción.

De Arriba y Cantero (2006), identifican dos tipos de mediación, una de ellas es la mediación de tipo personal, orientada a la negociación del discurso oral y una segunda, de tipo textual. En esta, se actúa sobre un texto determinado, sobre un lector desconocido y por tanto, no exige nuevas formas de negociación más allá del simple tratamiento de su estructura discursiva. De una u otra forma, este análisis exige un tratamiento más profundo, pues de acuerdo con los centros potenciadores de la enseñanza y su diversificación, no se estarían contemplando los intereses, necesidades y motivaciones de aprendizaje según lo señalado por los encuestados.

Un 64.4\% afirma conocer las siguientes microhabilidades de mediación lingüística como parte del proceso de facilitación del discurso escrito, entre las que encontramos: a) resumen, b) parafrasear e c) interpretar. Sin embargo, cabe preguntarse por qué aún no han logrado asumir una concepción sobre la facilitación 
de la lectura que no considere una visión adaptativa simplista y estática a ciertos grupos y perfiles, por sobre una concepción potente y potenciadora de texto accesible a un grupo amplio de personas que experimentan dificultades de comprensión.

Según esto, avanzar en materia de promoción de facilitación de la lectura o del discurso escrito, debe considerar que todo proceso de flexibilización del texto al perfil lingüístico y/o cognitivo del lector debe garantizar en términos discursos y lingüísticos: a) la adaptación debe tener en cuenta siempre el original, b) el adaptador aceptará la forma del texto literario original y c) el texto adaptado respetará las ideas y el estilo del autor.

En contraste Iewin (1986) identifica los factores que facilitan la comprensión en el estudiantado a partir de las estrategias de mediación que pueden disponer los docentes, entre las cuales figuran: a) utilizar referencias pronominales, como las relaciones semánticas del texto para influir en la comprensión, b)promocionar elementos conectivos inter-oracionales del texto (especialmente relaciones conjuntivas) parecen poseer un peso importante en la comprensión y c) enfatizar en las marcas sintácticas del texto, principalmente basadas en el modelo de información vieja/nueva, tales como construcciones de apertura y de preguntas contribuyen poderosamente a la comprensión.

Una de las manifestaciones más llamativas de los resultados arrojados por los docentes, es que un $25 \%$ de ellos señala no conocer los procesos de simplificación lingüística y filológica de textos literarios como elementos de promoción de la facilitación de la lectura a todos los colectivos de ciudadanos. No obstante, al consultarles si los procesos de mediación lingüística cobra especial relevancia cuando se presenta al mismo nivel que las demás actividades comunicativas, éstos en un $100 \%$ afirmaron estar totalmente de acuerdo.

Asimismo, un 25\% de los mismos, señaló estar en desacuerdo con que el proceso de mediación lingüística de cualquier texto literario es un proceso complejo, debido a que se debe captar el significado, la intención del mediador y el tipo de dificultades que genera a nivel discursivo, pragmático y cultural en relación al nivel de cualidad de los conocimientos previos de nuestros estudiantes. En contraste, un $50 \%$ de los docentes explicaron estar a favor de dicha afirmación.

Un 50\% de los encuestados, explica que en sus clases no logra integrar estrategias de mediación textual como recurso de accesibilidad al texto y a sus estructuras, sólo un $25 \%$ de ellos, señala que es una práctica habitual vinculada a su práctica pedagógica. En este contexto, el tipo de facilitación que mayormente describen los docentes es la mediación de tipo socio-pedagógica y de proximidad didáctico-formativa, los que en un $75 \%$ señalan su utilización en sus actividades formativas. 
La integración de la estrategia de mediación personal como medio de accesibilidad al proceso formativo y a las estructuras lingüísticas del texto en uso es utilizada por un $25 \%$ de los docentes, mientras que el $50 \%$ afirmo no conocer este procedimiento. Otro aspecto relevante, resulta de la intencionalidad pedagógica desarrollada por los profesores, quiénes afirman en un 50\% no considerar las necesidades, intereses y motivaciones formativas de mis estudiantes integró actividades de potenciación de la lectura desde la perspectiva de la mediación intralingüística. Sin duda, esto refleja una respuesta educativa desconectada de los centros potenciadores del aprendizaje, demostrando un cierto nivel de homogenización educativa.

No obstante, un $43.2 \%$ afirma que las adaptaciones realizadas por ellos y empleadas en sus clases, en términos discursivos y lingüísticos no logra valorar un texto para otros (no existe un fenómenos de alteridad, de reconocimiento del Otro y por el Otro al interior de la gestión de la enseñanza). Se observa además que un $30.1 \%$ demuestra debilidades vinculadas al tratamiento de los valores y unos elementos intrínsecos del texto (intencionalidad del autor, temas, tratamiento, estilo, entre otros). Sólo un $22.76 \%$ de los docentes conocen los criterios y las fórmulas que proporcionan el análisis pertinente de la frecuencia de palabras (IFP), así como las de marcadores discursivos destinados a evitar términos que imposibiliten que el mensaje llegue al receptor o bien, que sean ambiguas.

El $75 \%$ enfatiza que al momento de facilitar la lectura de un texto literario considera las características del interlocutor, del estudiantado y las palabras de modo que sean cercanas y comprensibles. Finalmente, un $25 \%$ de los mismos, señala que durante el proceso de mediación del texto literario como recurso didáctico, procuran mantener intacto el sustrato ideológico de este y mantener las motivaciones que orientaron la redacción de autor.

\subsubsection{El texto literario como recurso didáctico en la enseñanza del español como L2}

Desde el punto de vista del valor didáctico que adquiere el texto literario en el marco de la facilitación de la lectura en ELE, un $75 \%$ de los docentes señalan estar totalmente de acuerdo con que el texto literario se presenta como una valiosa herramienta de potenciación de la lectura, la imaginación y la creatividad en el aprendizaje de una segunda lengua.

El 25\% de los mismos, explica que el texto literario no permitiría potenciar la integración de las cuatro destrezas lingüísticas básicas, desde la adquisición consciente del significado en uso por parte de los estudiantes, no así un 50\% restante que señala que esta tipología textual representaría una fuente de enriquecimiento en activo del aprendizaje de las destrezas lingüísticas básicas asociadas al aprendizaje de una segunda lengua. 
Asimismo, un $75 \%$ de éstos, sostiene que la utilización del texto literario fomenta la interacción entre las representaciones previas de los estudiantes y los niveles de determinación que debemos realizar en el marco de la potenciación del comportamiento y de la comprensión lectora.

\subsection{Análisis de Resultados referidos a Escala Likert dirigida a Estudiantes de Español como L2}

\subsubsection{Gustos y Preferencias incidentes en el Proceso Lector}

Si bien el 13\% de los estudiantes encuestados, señala destinar más de 10 horas semanales de lectura en su L2, de lo cual se infiere que sus opciones, gustos y preferencias de lectura se encuentran sujetas a textos escasamente pertinentes a su realidad formativa y sociocultural. Un $62.5 \%$ explica que prefiere leer textos acorde a sus intereses, necesidades y motivaciones más que aquellos textos entregados en la clase. Esto nos lleva a pensar que la clase de español no responde a la heterogeneidad de los estudiantes, ni tampoco integra en su enfoque didáctico un sistema aumentativo que centre la enseñanza en el estudiante, como así en sus debilidades y fortalezas, impidiendo avanzar sobre la finalidad última del proceso lector en segundas lenguas como es la plena participación social.

Sólo el 25\% señala estar totalmente de acuerdo con la importancia de dedicar mayor parte de su tiempo libre a diversas actividades de lectura. No obstante, en Chile los espacios de diversificación social, cultural y educativa para colectivos de ciudadanos no hablantes de español, especialmente de procedencia haitiana; no han sido considerados en el marco de las políticas públicas de derechos lingüísticos y educativos, por lo que la respuesta continua siendo del tipo seudo-inclusiva e impertinente a sus manifestaciones lingüísticas.

\subsubsection{Evaluación de las Estrategias de Lectura para una enseñanza del español como L2 más oportuna}

Un 37.5\% de los estudiantes señala determinar la información general contenida en el texto al pasar la vista al pasar rápidamente por éste. Mientras que un $25 \%$ recurre a la técnica de lectura del primer y último párrafo para conformar una idea general sobre la información contenido en el texto, de lo cual se infiere que las vías de acceso al léxico y al texto, han sido escasamente potenciadas en relación al rendimiento y comportamiento lector. Asimismo, un 25\% señala estar totalmente de acuerdo que a través de la forma en que lograr captar la información del texto, es mediante el establecimiento de hipótesis y relaciones entre la información dispuesta en el texto de forma superficial.

Se observa que el $37.5 \%$ de los estudiantes no presentan un dominio efectivo de habilidades generales para enfrentar un texto, especialmente no recurren a la 
técnica de subrayado y organización de la información más relevantes desprendida de este. Un aspecto curioso es que el $25 \%$ y $37.5 \%$ de los aprendientes de español como L2, señalan integrar algo o parte de sus esquemas previos al momento de abordar un texto. Sin embargo, se desconoce debido a las características del instrumento la efectividad de dicha integración en tanto procedimientos cognitivas y de interacción textual.

\subsubsection{Procedimientos y Estrategias de aproximación al texto como recurso de aprendizaje autónomo en la enseñanza del español como L2}

Dentro de las principales estrategias de aprendizaje empeladas por los estudiantes haitianos para abordar un texto de tipo literario, se observa que un $37.5 \%$ no integra a su proceso lector estrategias de síntesis, resumen u organizadores gráficos para condensar los aspectos más relevantes y significativos de aquello que leen. Sólo un $25 \%$ subraya o marca palabras dentro del texto con la finalidad de verlas y así poder recordar más rápidamente la información.

Entre un $37.5 \%$ a un $50 \%$ de los encuestados, explica que sus hábitos de lectura se caracterizan principalmente por memorizar ideas o palabras, demostrando así un estilo estratégico de aprendizaje, debido a un $37.5 \%$ de los mismos, señala que al estudiar un texto leído en clases, se concentra en aquellas cosas que les tienden a parecer más importantes y que probablemente puedan ser preguntas de la prueba. Estos aprendientes en un 25\% describen aprender los temas leídos y tratados en clases con sus propias palabras en vez de memorizarlos al pie de la letra, mientras que sólo el 12.5 se encuentra totalmente en desacuerdo con esta actitud para el aprendizaje.

De acuerdo con el análisis de regresión lineal simple se comprobó que el valor predictivo de los esquemas previos y de la memoria operativa sobre el comportamiento lector de los estudiantes obteniendo un $88.6 \%$ de varianza en la comprensión lectora de aquellos aprendices avanzados; mientras que el nivel de memoria operativa contribuyó a explicar en un $77.9 \%$, independiente de la complejidad de los textos.

Si bien, los estudiantes más avanzados de español provenientes de Haití obtuvieron un $56.4 \%$ en la varianza asociada al rendimiento lector para la versión menos compleja (adaptación realizada para este estudio), mientras que su nivel de memoria operativa sólo alcanzó un $41.3 \%$ de frecuencia independiente del nivel de complejidad estructural y sintáctica de los textos.

Las interpretaciones por comparación múltiple demostraron que los estudiantes haitianos ubicados en un nivel de competencia B1 señalan dedicar un mayor porcentaje de horas de lectura en L2, no así los estudiantes ubicados en el nivel A1 y A2. Sin duda, los alumnos B1 evidencian un mayor repertorio de 
estrategias de procesamiento a la lectura que los ubicados en el tramo de competencia A1. Todo esto, nos invita a asumir en la gestión de respuestas oportunas en materia de facilitación de la lectura la relevancia de la competencia literaria.

\section{Conclusiones}

La facilitación del discurso escrito representa hoy un desafío importante en la gestión de respuestas educativas y sociales pertinentes a las demandas de todos los ciudadanos, incluidos en ellos, aquellos que no dominan el español a nivel funcional.

Entender el proceso de lectura y su aprendizaje, desde una perspectiva comunicativa y multidimensional de la misma, implica trasladar los factores de análisis desde los procesos perceptivos a factores de orden textual y estructural (Van Dijk, 1983), especialmente sobre los niveles de organización del significado relacionados entre sí, tales como el microestructural, el macroestructural y el superestructural.

La facilitación de la lectura se constituye como una herramienta potencialmente atractiva para superar las barreras asociadas al rendimiento y comportamiento lector, ya que compatibiliza un sistema puramente lingüístico y discursivo destinado a aproximar textos literarios y jurídicos personas con dificultades lectoras.

De acuerdo con Feuerstein, la experiencia de aprendizaje mediado y el rol del profesor como mediador consistiría en servir como una guía para modelar las interacciones que producirán mediante una interacción entre el sujeto de aprendizaje y de un agente que sirve de ayuda entre él y el ambiente (procesos formativosambientales).

La construcción de la comprensión de un discurso escrito adquiere un carácter multiestructural, debido a que la representación y significado articulado por el lector nos permite entender cómo se logra la coherencia local y la global, en contraste con factores del tipo de representación textual y situacional

Se identifica que en la actualidad las dimensiones epistémicas que sustentan el discurso de la legibilidad y la lecturabilidad en el campo de la didáctica de segundas lenguas, refieren únicamente a aspectos físicos y estructurales, desconociendo el potencial metodológico asociado a los factores sintácticos y filológicos de textos adaptados en su vertiente textual, por lo que representa una herramienta didáctica relevante para la formación de los futuros docentes de español al momento de enfrentar a los estudiantes a textos cada vez más desafiantes, no sólo desde su calidad sino que desde las habilidades cognitivas que permite estimular más oportunamente. 


\section{Bibliografía}

Acosta, L. (1989). El lector y la obra. Teoría de la recepción literaria. Madrid: Ed. Gredos.

Adams, J.M. (1970). Quels types de textes. París: Hachette.

Alonso, J., Mateos, M. (1985). “Comprensión lectora: modelos, entrenamiento y evaluación”, Revista Infancia y Aprendizaje, 31-32, 5-19.

Anula, A., Fernández-Lagunilla, M., Belichón, M., Revilla, A., Heras, L. (2005). Introducción al Quijote de la Mancha de Fácil Lectura. Madrid: SGEL.

Armstrong, T. (1999). Las inteligencias múltiples en el aula. Buenos Aires: Manantial.

Arroyo, R., Beard, R., Olivetti, M., Balpinar, Z., Silva, J. (2009). "Desarrollo intercultural de la composición escrita”, Revista de Educación Inclusiva, 2, (1), 103-121.

Arroyo, R. (2013). "Descripción de procesos en la composición escrita de estudiantes universitarios para un desarrollo multilingüe y tecnológico", Revista de Investigación Educativa, 31, (1), 167-184.

Bartlett, F. (1932). Remenbering: A study in experimental and social psycology. Cambridge: Cambridge University Press.

Belinchón, M., Igoa, J.M., Riviére, A. (2007). Psicología del Lenguaje. Investigación y teoría. Madrid: Trotta.

Bermeosolo, J. (2012). Psicología del lenguaje. Una aproximación psicopedagógica. Santiago: Ediciones PUC.

Beuagrande, R., Dressler, W. (1981). Introduction to Text Linguistics. London: Longman.

Benveniste, É. (2007). Problemas de Lingüística General. Tomo I. México: Siglo XXI.

Brown, G., Yule, G. (1990). Análisis del Discurso. Madrid: Visor Libros, S.L.

Bunge, M. (1980). Ciencia y Desarrollo. Buenos Aires: Editorial Sudamericana.

Bryant, P., Bradley, L. (1985). Problemas infantiles de lectura. Madrid: Alianza. 
Calero, A. (1997). Materiales curriculares para favorecer el acceso a la lectura en Educación Infantil. Madrid: Escuela Española.

Calero, A. (2010). Cómo mejorar la comprensión lectora. Estrategias para lograr lectores competentes. Madrid: Wolters Kluwer.

Cantero, F.J. (1998). "conceptos clave en lengua oral", en Mendoza, A. (Coord.): Conceptos clave en didáctica de la lengua y la literatura. Barcelona: Horsori.

Cardona, M. (2002). Introducción a los Métodos de Investigación en Educación. Madrid: Editorial EOS.

Cassany, D. (1993). “Los procesos de redacción”, Cuadernos de Pedagogía, 216, 8284.

Cassany, D., Luna, M., Sanz, G. (1994). "La cultura de la escritura: planteamientos didácticos", en AA.VV.: Aspectos didácticos de la lengua y literatura, 8, Zaragoza: ICE, 11-46.

Consejo de Europa. (2002). Marco Común Europeo de Referencia. Madrid: Instituto

Cervantes. [en línea]. Disponible en http://cvc.cervantes.es/ensenanza/biblioteca_ele/marco/cvc_mer.pdf

[Consultado: 05 de septiembre, 2013].

Cuetos, F. (2011). Psicología de la lectura. Madrid: Wolters Kluwer.

CVC. (2013). Diccionario de Términos Claves en ELE. Madrid: Instituto Cervantes. [en línea]. Disponible en http://cvc.cervantes.es/ensenanza/biblioteca_ele/diccio_ele/ [Consultado: 20 de noviembre, 2013].

De Arriba, C. (2003). La mediación lingüística en la enseñanza de lenguas extranjeras. Tesis doctoral. Dep. de Didáctica de la Lengua y la Literatura. Universidad de Barcelona.

De Arriba, C., Cantero, J.F. (2006). "La mediación lingüística en la enseñanza de lenguas", Revista Didáctica. Lengua y Literatura, 16, (2), 9-21.

Defior, S. (2005). "La conciencia fonológica y la adquisición de la lectoescritura", Revista Infancia y Aprendizaje, 67 (4), 91-113.

Denyer, M. (1988). La lectura: una destreza pragmática y cognitivamente activa. Madrid: Universidad Antonio de Nebrija.

De Vega, M. (1990). Lectura y comprensión. Una perspectiva cognitiva. Madrid: Alianza. 
Fernández, S. (1999). Interlengua y análisis de errores en el aprendizaje del español como lengua extranjera. Madrid: Eldesa S.A.

Freinet, C. (1973). El texto libre. Barcelona: Laia.

Follari, R. (2007). Epistemología y sociedad. Acerca del debate contemporáneo. Buenos Aires: HomoSapiens.

Fons, M. (2004). Leer y escribir para vivir: alfabetización inicial y uso real de la lengua escrita en el aula. Barcelona: Graó.

García, J., González, D. (2000). Dificultades de Aprendizaje e Intervención Psicopedagógica. Madrid: EOS.

Gil, J.M. (2001). Introducción a las Teorías Lingüísticas del Siglo XX. Santiago: RIL Editores.

González, R. M. (2005). Poder escribir: programa de entrenamiento en los procesos cognitivos de la escritura. Libro del Educador. Madrid: EOS.

Giddens, Turnner (2010). La teoría social hoy. Madrid: Alianza.

Gispert, D., Ribas, L. (2012). Alumnado con dificultades en el aprendizaje de la lectura. Madrid: Graó.

Gregorio, R. (2003). Metodología de la Investigación Cualitativa. Málaga: Ediciones Aljibe.

Halliday, M.A.K., Hassan, R. (2000). Language, Context and Text: Aspects of Language in a Social Semiotic Perspective. Cambridge: CUP.

Hernández, R., Fernández, C., Baptista, P. (2003). Metodología de la Investigación. Ciudad de México: McGraw-Hill.

Hymes, D. H. (1971), “Acerca de la competencia comunicativa”, en LLOBERA et al. (1995), Competencia comunicativa. Documentos básicos en la enseñanza de lenguas extranjeras. Edelsa, Madrid, 27-47.

Inchaurralde, C., Vázquez, I. (2000). Una introducción cognitiva al lenguaje y la lingüística. Zaragoza: Mira.

Irwin, J. (1986) Teaching reading comprehension processes. Englewood Cliffs. N.J.: Prentice-Hall

Johnson, P. (1981). "Effects on reading comprehension of language complexity and cultural background of a test", Tesol Quarterly, 15,(2), 169-181. 
Landívar, A. (2013). Neuroeducación. Educación bajo la lupa de María Montessori. Córdoba: Brujas.

Kintsch, W. (1988). "The role of knowledge in discourse comprehension: a construction-integration model”, Psychological Review, 162-182.

Lebrero, M.P. (1988). Cómo y cuándo enseñar a leer y escribir. Madrid: Síntesis.

Llobera, M. (1995). Competencia comunicativa. Documentos básicos en la enseñanza de lenguas extranjeras. Madrid: Visor.

Mendoza, A. (2001). El intertexto lector. Cuenca: Universidad de Castilla-La Mancha.

Mendoza, A. (2010). Didáctica de la lengua y la literatura. Madrid: Pearson.

Montessori, M. (1934). Psico Arithmética. Barcelona: Casa Editorial Araluce.

McMillan, J.H., Schumacher, S. (2007). Investigación Educativa. Una introducción conceptual. Madrid: Pearson.

Pérez, G. (1998). Investigación Cualitativa: Retos e interrogantes. Vol. I y II. Madrid: La Muralla.

Pinker, S. (1995). El instinto del lenguaje. Cómo crea el lenguaje la mente. Madrid: Alianza.

Ocampo, A. (2014). "En busca del saber pedagógico y del saber epistémico de la educación inclusiva: ideas sobre un enfoque paradigmático en evolución" Actas del Congreso Internacional Infancia en Contextos de Riegos, AICE: Huelva, 2650-2663.

OCDE. (2009). Iberoamérica en PISA 2009. Informe regional. Madrid: Santillana

Orlandi, E. (2012). Análisis del discurso. Principios y procedimientos. Santiago: LOM.

Schmidt, S. (1984), "Empirische Literaturwissenschaft in der Kritik", en SPIEL, 3, (2), 291-332.

Solé, I. (1993) "Lectura: ¿placer?, ¿obligación? Cómo se adquiere el gusto por la leer: ¿Se obliga?, ¿Se aconseja? ¿Se recomienda?”. Primeras noticias. Literatura Infantil y Juvenil, 118, (3), 32-38.

Solé, I. (2012). "Competencia lectora y aprendizaje", Revista Iberoamericana de Educación, 59, 43-61. 
Singer, M. (1990). Psychology of Language: An introduction to sentence and discourse processe. N.J.:LEA.

Sousa, V., Driessnack, M.,Costa, I. (2007). Revisión de los diseños de investigación relevantes para la enfermería: métodos mixtos y múltiples, Revista LatinoAmericana de Enfermagem, 15, (5), 179-182.

Snow, C.E. (2001). Reading of Understading. CA: RAND.

Stone Wiske, M. (2006). La Enseñanza para la Comprensión: vinculación entre la investigación y la práctica. Buenos Aires: Paidós.

Swartz, S. (2012). Cada niño un lector. Estrategias innovadoras para enseñar a leer y escribir. Santiago: Ediciones PUC.

Van Dijk, T. (1982). La ciencia del texto. Barcelona: Paidós.

Van Dijk, T. (1993). Texto y contexto. Semántica y pragmática del discurso. Madrid: Cátedra.

Van Dijk, T.y Kintsch, W. (1978). Toward a model of text comprehension and production. Psychological Review, 85,(5), 362-394.

Van Esch, K. (2010). "La comprensión lectora del español como lengua extranjera: necesidades comunicativas, objetivos y métodos de enseñanza-aprendizaje", Revista Marco ELE 11, (1), 274-303.

Widdowson, H.G. (1990). Aspects of language teaching. Oxford: O.U.P.

Widdowson, H.G. (1995). "Conocimiento de la lengua y habilidad para usarla", en Llobera, M. (Coord). Competencia comunicativa: documentos básicos en la enseñanza de lenguas extranjeras, Madrid: Eldesa, 83-90.

Wolf, D. (1985). "Verstehensprozesse in einer zweiten Sprache". In: Studium Linguistik, 17, (18), 162-174.

Zemelman, H. (2012). Sujeto, existencia y potencia. Barcelona: Anthropos. 